\title{
Elizabethkingia meningoseptica Infection in a Neonate: Case Report
}

\author{
Sandhra Davis ${ }^{1, *}$, Roshni Acha Biju², Ganga Sanal ${ }^{2}$ \\ ${ }^{1}$ St. James' College of Pharmaceutical Sciences, Chalakudy, Thrissur, Kerala, INDIA. \\ ${ }^{2}$ Nazareth College of Pharmacy, Othera, Pathanamthitta, Kerala, INDIA.
}

\begin{abstract}
Elizabethkingia meningoseptica is a gram-negative bacteria associated with multiple nosocomial infections normally present in hospital environments. In particular, preterm neonates are more susceptible to develop serious infections. This is a case of E. meningoseptica septicemia that was detected early in an extremely premature low birth weight infant with other co morbidities, He was successfully treated with a combination of levofloxacin and Meropenem. The spread of infection was controlled with no other reported cases. Accurate and early detection with appropriate sensitivity testing is mandatory to reduce morbidity in patients with E. meningoseptica.
\end{abstract}

Key words: Elizabethkingia meningoseptica, Nosocomial infections, Preterm infant, Active surveillance.

\section{INTRODUCTION}

Elizabethkingia meningoseptica is a pervasive Gram-negative bacillus bacteria, belonging to the family Flavobacteriaceae, widely distributed around including the hospital envoirment. ${ }^{1}$ It usually results in meningitis and sepsis in newborns. Bacteremia and pneumonia are the other prominent causes in neonates. Premature infants are affected the most as they are a major risk factor for E. meningosepticum infection. ${ }^{2}$ The diagnosis is based on cultures obtained from blood and CSF samples.

Immunosupression, prolonged hospital stay, underlying co morbid medical conditions, central venous catheter indwelling or other intrusive devices are the risk factors for acquiring this infection. The long term use of broad spectrum antibiotics is another significant contributing factor. The improper antibiotic selection for initial empirical therapy leads to therapy failure which remains as a challenge to physicians. ${ }^{3,4}$ Early detection of organism with significant susceptibility reports is necessary to reduce mortality in E. meningoseptica infected patients.

\section{CASE REPORT}

A preterm female neonate was born to 29 year old primigravida vaginal delivery after 24 weeks gestation with birth weight of 460 g. The infant had a weak cry at birth; she was electively intubated and transferred to NICU and blood cultures taken at birth showed no growth. Baby had hyperbilirubinemia on day 2 and phototherapy was given.

Laboratory investigations at birth shows total leukocyte count $-53.3 \mathrm{~K} / \mathrm{uL}$, neutrophil count $-64 \%$, lymphocytes -20\%, eosinophil- 3\%, monocyte- 4\%, C-reactive protein $-7.9 \mathrm{mg} / \mathrm{l}$, total Bilirubin $-3.3 \mathrm{mg} / \mathrm{dl}$. ECHO done on day 3 showed no evidence of patent ductus arteriosus. Baby did not require inotropic support and was started on prophylactic paracetamol.
DOI: 10.5530/ijopp.14.4.64

Address for correspondence: Dr. Sandhra Davis, Pharm D, St. James' College of Pharmaceutical Sciences, Chalakudy-680307, Thrissur, Kerala, INDIA. Phone no: +917356238151 Email id: sandradavis19july@ gmail.com

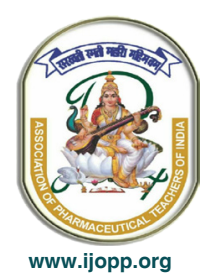


On day 6 post birth, baby started experiencing fever with TLC count $46.4 \mathrm{~K} / \mathrm{uL}$ and CRP is 20 . She developed poor feeding and desaturation followed by bradycardia requiring resuscitation. Baby also had hyponatremia, hypokalemia, hypocalcemia and metabolic acidosis. Septic screen was sent and empirical antibiotic treatment with IV ampicillin and amikacin was started along with prophylactic inj.fluconazole. Septic screen was positive and ampicillin was switched to piperacillin -tazobactam. Blood culture was sterile initially on day 8 th. However the symptoms persisted and frequent blood culture on day $16^{\text {th }}$ isolated Elizabethkingia meningoseptica by a VITEK 2 system. The isolate showed sensitivity toward levofloxacin (MIC $0.5 \mu \mathrm{g} / \mathrm{ml}$ ), ciprofloxacin (MIC $1 \mu \mathrm{g} / \mathrm{ml}$ ), co-trimoxazole (MIC $<=20 \mu \mathrm{g} / \mathrm{ml})$, minocycline $(<=1 \mu \mathrm{g} / \mathrm{ml})$ and resistant to piperacillin tazobactam. IV piperacillintazobactam was stopped and IV meropenem and IV levofloxacin was started and continued for14 days and showed marked clinical improvement.

The infant was consulted by a neurosurgeon for CSF drainage and neuropsychiatric monitoring to avoid late complications. Epidemiological data indicated this as an isolated case. No source of infection was identified.

Additional specific intensive care interventions contributed to case management, including supplemental oxygen with high-flow nasal cannula, intravenous hydrocortisone infusion.

\section{DISCUSSION}

Elizabethkingia meningoseptica belongs to the Chryseobacterium genus. It is a non-fastidious, nonmotile, aerobic, oxidase -positive and catalase -positive, Gram-negative bacillus reported by Elizabeth. O. King in $1959 .{ }^{5}$ Reports have shown existence of this organism in colonizing sink basins and taps; chlorinetreated water, in ventilator tubing; oxygen humidifiers; infusion pumps; intravenous fluid stands; hand soap dispensers; cabinets, bedside shelves, drawers and ; and stethoscopes. ${ }^{6}$

Preterm infants, immunocompromised, and those on antibiotics or in critical care units are most vulnerable to E. meningoseptica infections. It can lead to bacteremia, septicemia, and meningitis. Apnea, bradycardia and respiratory distress are the key clinical features of neonatal sepsis, observed in the present study. The variables of virulence that are responsible for invasive E. meningoseptica infection have not yet been thoroughly elucidated. Premature birth is a relevant risk factor for nosocomial infections in infants and neonates who weighed less than $2.5 \mathrm{~kg}$ at birth. ${ }^{7}$

E. meningoseptica though being a gram-negative bacillus, resistant to gram-negative (beta-lactam antibiotics, aminoglycosides, chloramphenicol and tetracyclines) antibiotics, but is susceptible to Gram positive agents (clindamycin, rifampicin, trimethoprimsulfamethoxazole, erythromycin, vancomycin, and quinolones). ${ }^{8}$ This could lead to inappropriate antibiotic selection in initial therapy and result in treatment failures. Early diagnosis and proper infection control could improve patient outcome. Active infection control measures like regular inspection of the hospital water tanks, water surveillance, and hyper chlorination of the water might be helpful to prevent the infection with these challenging bacteria. ${ }^{9}$ Failure in recognizing this pathogen could lead to an inadequate antibiotic coverage and treatment failure. In order to minimize it, clinicians need to make timely diagnosis and sensitivity testing to ensure proper treatment.

\section{CONCLUSION}

There is an emerging trend of Elizabethkingia meningoseptica infections among premature newborns and immunocompromised patients, priorly owing to lack of proper hygiene in hospital setting and bacterial resistance. Good communication between physicians and laboratory staff is essential for the proper management of patients. Rapid diagnosis and appropriate therapy of nosocomial infections will help to reduce the incidence of such infections in a hospital setting.

\section{REFERENCES}

1. Bhat KS, Priya R, Krishnan L, Kanungo R. Elizabethkingia meningoseptica bacteremia in a neonate: A case report and mini-review of the literature. J Curr Res Sci Med. 2016;2(1):42-5. doi: 10.4103/2455-3069.184130.

2. du Moulin GC. Airway colonization by Flavobacterium in an intensive care unit. J Clin Microbiol. 1979;10(2):155-60. doi: 10.1128/jcm.10.2.155-160.1979, PMID 511985.

3. Sarma S, Kumar N, Jha A, Baveja U, Sharma S. Elizabethkingia meningosepticum: an emerging cause of septicemia in critically III patients. J Lab Physicians. 2011;3(1):62-3. doi: 10.4103/0974-2727.78575, PMID 21701671.

4. Lin PY, Chu C, Su LH, Huang CT, Chang WY, Chiu CH. Clinical and microbiological analysis of bloodstream infections caused by Chryseobacterium meningosepticum in nonneonatal patients. J Clin Microbiol. 2004;42(7):3353-5. doi: 10.1128/JCM.42.7.3353-3355.2004, PMID 15243115.

5. Centers for Disease Control and Prevention (CDC) about Elizabethkingia; Updated March 30 2016. Available from: https://www.cdc.gov/elizabeth-kingia/ about/index.html [cited 8/9/2021].

6. Güngör S, Ozen M, Akinci A, Durmaz R. A Chryseobacterium meningosepticum outbreak in a neonatal ward. Infect Control Hosp Epidemiol. 2003;24(8):613-7. doi: 10.1086/502261, PMID 12940584. 
7. McQuiston J. Deadly midwest outbreak of Elizabethkingia. Medscape. Updated April 1 2016;April.

8. Tak V, Mathur P, Varghese P, Misra MC. Elizabethkingia meningoseptica: an emerging pathogen causing meningitis in a hospitalized adult trauma patient.
Indian J Med Microbiol. 2013;31(3):293-5. doi: 10.4103/0255-0857.115653, PMID 23883720.

9. Hung PP, Lin YH, Lin CF, Liu MF, Shi ZY. Chryseobacterium meningosepticum infection: antibiotic susceptibility and risk factors for mortality. J Microbiol Immunol Infect. 2008;41(2):137-44. PMID 18473101. 